\title{
An artifact in the use of brain stimulation with shock-motivated behavior'
}

\author{
J. S. Sehwartzbaum and Peter J. Donoviek \\ UNIVERSITY OF WISCONSIN
}

\begin{abstract}
Abstraet
The effects of continuous electrostimulation (ICS) of the septal area on passive-avoidance behavior were investigated under conditions in which grid-shock and ICS were either isolated or confounded. Such artifactual interactions can have major behavioral consequences. Brain stimulation induced by a grid-shock can effectively counteract the suppressive effects of the shock.
\end{abstract}

\section{Introduetion}

In studies of shock-motivated behavior that have employed electro-stimulation of the brain, investigators have shown varying degrees of concern with regard to the possible interactions between grid-shock and ICS circuits (Brady \& Conrad, 1960; Goddard, 1964; Kasper, 1964), i.e., inadvertent stimulation of the brain when $\mathrm{S}$ is shocked peripherally. There is little evidence about the magnitude of such effects, nor is it clear what their behavioral consequences might be. In this study, we sought to examine the generality of findings reported recently by Kasper (1964) dealing with the attenuating effects of continuous stimulation of the septal area on "passive-avoidance" behavior. In the context of this work, we examined the behavioral consequences of confounding the grid-shock circuit with the ICS circuit.

\section{Method}

The Ss in the major study were 14 male rats of the Sprague-Dawley strain, approximately $300 \mathrm{gm}$ in bodyweight. All were implanted with bipolar stainless steel electrodes (.010 in) in the septal area as illustrated by the inset in Fig. 1. In 10 of the Ss, cross-talk between grid-shock and ICS circuits was prevented by means of a relay mounted in the test enclosure that cut out the cable leading to the ICS equipment whenever $S$ was shocked peripherally. In five of these Ss (Group E), the septal area was stimulated continuously during tests of passive avoidance (except during actual occurrence of grid-shock). The other five Ss (Group C) served as nonstimulated controls. In the remaining four Ss, grid-shock was allowed to interact with ICS circuit by omitting operation of the relay in the test enclosure. Two of these Ss received stimulation of the septal area, comparable to Group E, while the other two received control treatment.

Stimulation was supplied from a Grass S-4 (with SIU-4B isolation unit) or SD-5 in the form of $20 \mu$ a, 100 cps "biphasic" pulses, each $0.5 \mathrm{msec}$. in duration (Kasper, 1964). Intensity of current was monitored by measuring the voltage drop across a $10 \mathrm{~K}$ resistor, using the differential inputs to a Tektronix 502A oscilloscope (each input 1 megohm with respect to ground).
The leads which ran from the control equipment to the test enclosure were $15 \mathrm{ft}$ long, low capacitance, and were shielded.

Initially all Ss were placed on a food-deprivation schedule (22 gm /day) and were trained in two lever boxes to press a bar for food ( $45 \mathrm{mg}$ pellet) programmed on a 30-sec. VI schedule (Schwartzbaum, Thompson \& Kellicutt, 1964). Electrodes were then implanted and Ss allowed five to six days to recover. Two additional sessions, each $20 \mathrm{~min}$. in duration, were then administered. In the last session, 5-min. periods of continuous stimulation were alternated with 5-min. periods of no stimulation to assess effects on baseline performance. None was observed in any S.

Tests of passive avoidance were then run for three 20-min. sessions. Responses in the first 5-min. periods of each session were never punished. During the remainder of each session, bar presses were punished intermittently on a 2-min. F1 schedule. The first response after each initial 5-min. period was punished and initiated the F1 contingency. Shock was delivered through grid floor-bars from a Grason-Stadler unit (E 1064GS) set at $0.8 \mathrm{ma}(\mathrm{AC})$ for $0.5 \mathrm{sec}$. At the conclusion of these tests, Ss were trained to self-stimulate with ICS (0.5 sec. pulse train) and the reward threshold was approximated by the method of limits.

\section{Results}

As shown in Fig. 1 (upper section), continuous stimulation of the septal area had no effect on passive avoidance when the shock circuit and ICS circuit were not confounded. Ss typically took two to three shocks before fear was evident and bar pressing was suppressed. Group E averaged 5.0 shocks (range 3 to 8 ) for the three sessions, while Group C averaged 4.8 shocks (range 3 to 7 ). Although these findings disagree with the earlier data (Kasper, 1964), it should be emphasized that behavioral test conditions differed markedly. In support of her findings, Group E showed significantly less defecation than did Group C $(p<.025$ by $U$ test) and, despite evidence of fear, made fewer freezing responses. These latter findings are similar to those obtained with lesions of the septal area (Schwartzbaum \& Spieth, 1964).

The lower section of Fig. 1 shows clearly that cross-talk between grid-shock and ICS circuits, regardless of the concurrent use of ICS, is a variable of major significance. Although response rates fluctuated somewhat in the course of testing, these Ss averaged 20 shocks (range 17 to 24 ) and typically showed no 


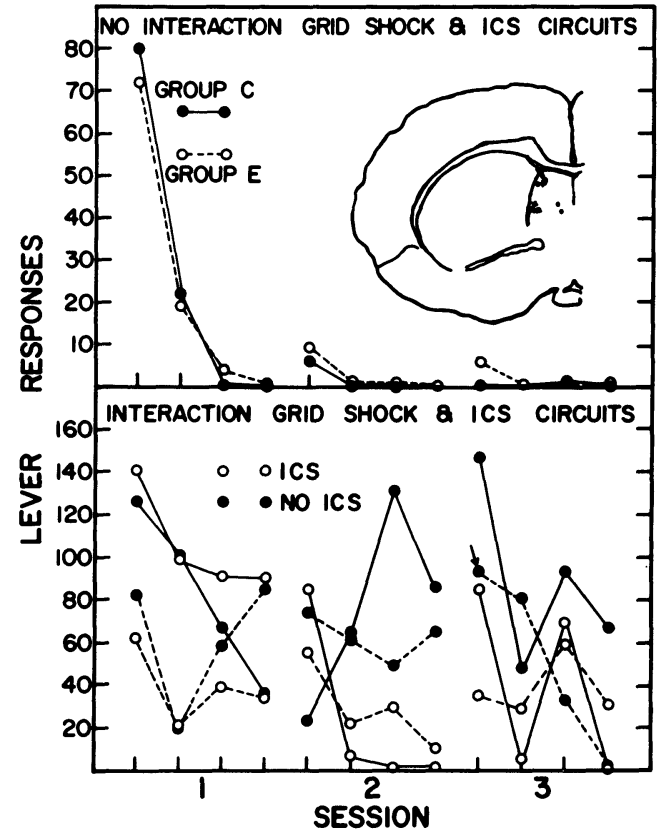

Fig. 1. Responses during successive 5-min. periods of each session (mean values, upper section; individual values, lower section). Insert shows placement of electrodes for Ss in Group E (dots) and for Ss exposed to grid-shock-ICS interaction (crosses).

consistent suppression nor evidence of fear. In one of the Ss (denoted by arrow in Fig. 1), the stimulation leads and shield were cut out at the control end of the circuit in session three. This animal then gradually suppressed responding. Oscilloscope readings on these Ss showed that the grid-shock induced 50-75 $\mu$ a AC flow of current through the ICS circuit. Since self-stimulation thresholds ranged from $50 \mu$ a upward, it is likely that, despite differences in waveform, the induced brain stimulation was positively rewarding. This effect of the stimulation may have counteracted the aversive properties of the grid-shock. Indeed, low reward thresholds seemed to relate to maximal behavioral effects from induced brain stimulation; in two instances, the gridshock produced a mild overt seizure. We have observed similar phenomena with hippocampal placements where reward thresholds were again low. One S pressed the bar repeatedly despite continuous punishment of the response. But unlike the type of phenomenon described by Brady \& Conrad (1960), we have not been able to block passive avoidance by controlled rewarding ICS to the septal area, when delivered either 2-sec. after each grid-shock or together with each food reward on the 30-sec. VI, i.e., when shock and ICS circuits were not confounded.

While we do not wish to go into any detailed explanation of the interaction phenomenon, two features about our ICS circuit may be noted. First, it offered about the same impedance as existed across the tips of the electrodes. Thus, any differences in AC potential induced by the grid shock across the electrodes would generate a flow of current into the parallel ICS circuit. Secondly, capacitance between the leads and shield of the connecting cable (shield was grounded) allowed some leakage of current from shock circuit to ground; input to the oscilloscope was less important in this respect. These and other factors should be carefully considered in employing brain stimulation and shock.

References

BRADY, J. V., \& CONRAD, D. G. Some effects of limbic system self-stimulation upon conditioned emotional behavior. J. comp. physiol. Psychol., 1960, 53, 128-137.

GODDARD, G. V. Amygdaloid stimulation and learning in the rat. J. comp. physiol. Psychol., 1964, 58, 23-30.

KASPER, P. Attenuation of passive avoidance by continuous septal stimulation. Psychon. Sci., 1964, 1, 219-220.

SCHWARTZBAUM, J. S., \& SPIETH, T. M. Analysis of the responseinhibition concept of septal functions in "passive-avoidance" behavior. Psychon. Sci., 1964, 1, 145-146.

SCHWARTZBAUM, J. S., THOMPSON, J. B., \& KELLICUTT, M. H. Auditory frequency discrimination and generalization following lesions of the amygdaloid area in rats. J.comp. physiol. Psychol., $1964,57,257-266$.

\section{Note}

1. Supported in part by grants, M-4686 and MH 10801, from the United States Public Health Service, National Institute of Mental Health, and by the Research Committee of the Graduate School from funds supplied by the Wisconsin Alumni Research Foundation. 